\title{
Reduced-complexity representation of the human arm active endpoint stiffness for supervisory control of remote manipulation
}

The International Journal of Robotics Research 2018, Vol. 37(1) 155-167 (C) The Author(s) 2017 Reprints and permissions: sagepub.co.uk/journalsPermissions.nav DOI: $10.1177 / 0278364917744035$ journals.sagepub.com/home/ijr

\author{
Arash Ajoudani', Cheng Fang ${ }^{2}$, Nikos Tsagarakis², and Antonio Bicchi ${ }^{3,4}$
}

\begin{abstract}
In this paper, a reduced-complexity model of the human arm endpoint stiffness is introduced and experimentally evaluated for the teleimpedance control of a compliant robotic arm. The modeling of the human arm endpoint stiffness behavior is inspired by human motor control principles on the predominant use of the arm configuration in directional adjustments of the endpoint stiffness profile, and the synergistic effect of muscular activations, which contributes to a coordinated modification of the task stiffness in all Cartesian directions. Calibration and identification of the model parameters are carried out experimentally, using perturbation-based arm endpoint stiffness measurements in different arm configurations and cocontraction levels of the chosen muscles. Consequently, the real-time model is used for the remote control of a compliant robotic arm while executing a drilling task, a representative example of tool use in environments with constraints and dynamic uncertainties. The results of this study illustrate that the proposed model enables the master to execute the remote task by modulation of the directions of the major axes of the endpoint stiffness ellipsoid and its volume using natural arm configurations and the cocontraction of the involved muscles, respectively.
\end{abstract}

\section{Keywords}

Human impedance modeling, teleimpedance control, remote manipulation, telerobotics

\section{Introduction}

Execution of a remote manipulation task in a master-slave teleoperation setup is often subject to dynamic uncertainties and jitter. Both issues result in an undesirable behavior of the slave robot and reduced efficiency in physical interactions with the object or the environment (Hannaford and Ryu, 2002). In an attempt to overcome the limitations of conventional teleoperation interfaces, the concept of teleimpedance control has been introduced (Ajoudani et al., 2012b), with several interaction scenarios in support of the efficacy of this control concept (e.g., see Hocaoglu and Patoglu, 2012).

Teleimpedance consists of replicating the desired motion trajectories and corresponding stiffness profiles of the operator by the compliant slave robot in real time. This is to enable the operator to modulate the task interaction forces between the robot and the environment while avoiding the closed-loop stability issues raised by traditional bilateral teleoperation interfaces. While the human movements can be tracked using accurate and cost-efficient external devices, the current literature lacks appropriate and computationally efficient methods for real-time estimation of the limb impedance. Especially concerning complex manipulation tasks that demand and explore the full capacity of human arm dynamics, traditional techniques for the estimation of the human joint torque and stiffness trajectories require several stages, from the measurement of biosignals to the musculoskeletal kinematics and dynamics.

It is well known that human beings modulate their limb endpoint viscoelastic properties in different ways. One approach to achieve this is by cocontracting muscle groups acting on the limb (Gribble et al., 2003). Alternatively, it can be performed through adaptation in the sensitivity of reflex feedback (Akazawa et al., 1983) or selective control of the limb configuration (Trumbower et al., 2009). Traditionally, the combined effect of these stiffness modulation mechanisms at the arm endpoint is explored by applying position

${ }^{1}$ Human-Robot Interfaces and Physical Interaction $\left(\mathrm{HRI}^{2}\right)$ Laboratory, Istituto Italiano di Tecnologia, Italy

${ }^{2}$ Humanoids and Human-Centred Mechatronics Laboratory, Istituto Italiano di Tecnologia, Italy

${ }^{3}$ Soft Robotics for Human Cooperation and Rehabilitation Laboratory, Istituto Italiano di Tecnologia, Italy

${ }^{4}$ The Interdepartmental Research Center "E. Piaggio", Faculty of Engineering, University of Pisa, Italy

Corresponding author:

Arash Ajoudani, Human-Robot Interfaces and Physical Interaction $\left(\mathrm{HRI}^{2}\right)$ Laboratory of the Istituto Italiano di Tecnologia, Via Morego 30, 16163, Genova, Italy.

Email: arash.ajoudani@iit.it 
(force) perturbations to the hand, and probing the restoring force (displacement response) profile (Mussa-Ivaldi et al., 1985; Perreault et al., 2002). This is usually followed by an offline postprocessing phase to estimate the impedance parameters. Applications of such methods in the estimation of dynamic impedance profiles in multijoint arm movements have been extensively investigated (Franklin et al., 2003).

Despite the popularity of perturbation-based approaches, their real-time applications result in an inconvenient and often impossible operation, owing to the interference of external disturbances with the hand trajectories. As a consequence, other avenues of research seek for a more suitable human-machine interface that is particularly beneficial for real-time applications (Ajoudani et al., 2012b; Howard et al., 2013; Osu and Gomi, 1999; Shin et al., 2009). In this direction, owing to the existence of high correlations between muscle activations, muscular force, and joint torque profiles, a large portion of the related literature utilizes electromyography (EMG) signals to account for the real-time tracking of the arm joint or endpoint stiffness profile. While in a fixed arm configuration, such a modeling turns out to be straightforward, owing to the linear association between the EMG and arm endpoint stiffness profiles (Selen et al., 2005), the whole arm workspace stiffness estimation appears to require a complex modeling of the musculoskeletal system (Lloyd and Besier, 2003; Shin et al., 2009). This, however, contrasts with several studies on human motor behavior, which suggest that the central nervous system solves for this complexity and redundancy in an elegant, effective, and rather coordinated manner (Kawato, 1999; Turvey, 2007).

Observations in human neuromotor control of the arm endpoint stiffness suggest that, owing to (i) the major contribution of the limb geometry to efficient modifications in the orientation of the endpoint stiffness ellipsoid, (ii) the ergonomic efficiency of postural adjustments in comparison with cocontractions, and (iii) the existence of crossjoint muscles in limbs, human beings tend to maximize the use of limb postures to realize a desired endpoint stiffness direction (Milner, 2002). Concurrently, cocontractions appear to mostly contribute to modifications in size, rather than orientation of the stiffness ellipsoid (Ajoudani, 2016; Milner, 2002). A reason for this is deemed to be the involvement of the arm muscles in a synergistic fashion (Castellini et al., 2014; Ison and Artemiadis, 2014a,b; Turvey, 2007), which contribute to coordinated variations in the diagonal (joint stiffness) and off-diagonal (owing to the existence of cross-joint muscles) components of the joint stiffness matrix (Buchanan et al., 1986; Jamison and Caldwell, 1993; Osu and Gomi, 1999; Van Zuylen et al., 1988).

On these bases, we explore the role of arm geometry and muscular contraction in modifications in direction and volume of the arm endpoint stiffness ellipsoid, respectively. To do so, we investigate the configuration-dependent properties of the joint and Cartesian stiffness profiles in human beings through the muscle and arm Jacobians. With the purpose of using a reduced number of tracking points on the human arm to account for the arm kinematics, a generalized arm triangle model is introduced and used to estimate the joint angles and the muscle and arm Jacobians. In comparison with the alternative descriptions, the generalized human arm triangle in this work is able to express the arm posture in a more accurate way by considering physiological geometric constraints between segments (e.g., the elbow joint does not allow for abduction-adduction rotation) and the whole skeleton model of the human arm. Next, a cocontraction index is defined and experimentally identified to implement the volume-adjusting component of the arm endpoint stiffness.

With the models identified, the endpoint stiffness of the human arm is estimated by tracking the arm kinematics and the volume-adjusting component in real time. This enables the master to modify the direction of the endpoint stiffness ellipsoid by changing the arm posture in an intuitive manner, while being capable of adjusting its volume by increasing the cocontraction of the dominant shoulder and elbow muscles. As a result, teleoperated tasks that require significant modulation of the endpoint stiffness and force can be executed naturally. In this study, as a representative example of the tasks with environmental constraints and dynamic uncertainties, we consider the drilling of hard objects that are placed in different locations and orientations within the robot workspace (see Figure 1). To accomplish the task, the end-effector force (as a consequence of the stiffness geometry and volume modulation) must be remotely controlled to achieve a high interaction force in the direction of drilling, while avoiding the generation of unnecessarily high interaction forces in other directions or other phases of the task (e.g., sliding on the surface) that might be caused by involuntary movements of the human hand. Such undesired forces can cause damage to the robot, tool, or environment, especially when the drill penetrates the hard object. This behavior is way beyond the interaction capabilities of rigid robots, i.e., those operated in position control mode or replicating a constant joint or Cartesian impedance profile.

Needless to say, any reduced-complexity representation of the highly complex human neuromotor system is certainly subject to modeling uncertainties and inaccuracies. For instance, we assume that both the robot and the human operator are constrained to stand in place and that the operator arm moves in relatively low endpoint acceleration profiles. The reason for this is to simplify the tracking of the human configuration, since the human whole-body poses (e.g., using legs) can contribute to further modifications in the geometry of the endpoint stiffness ellipsoid. In addition, low acceleration profiles of the operator arm reduce the effect of inertia mismatch between the human arm and the robot in the case of an impact. The main purpose of this research is to provide computationally efficient and 


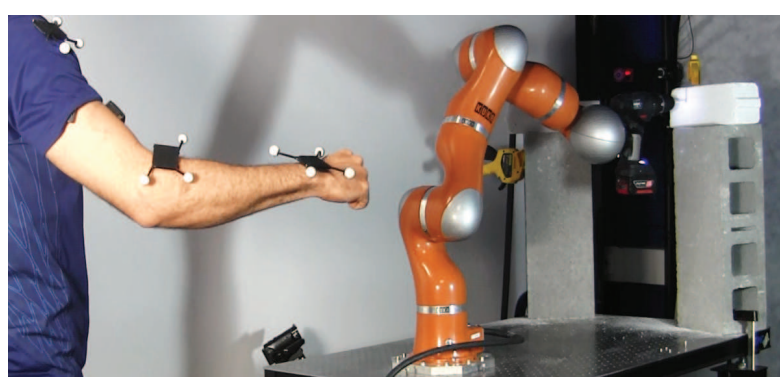

Fig. 1. Experimental setup for remote drilling of hard objects (bricks) in different positions and orientations of the robot workspace. To accomplish the task, the operator must adjust the endpoint stiffness profile of the robot end-effector to achieve the task-required interaction force profile in different phases of the task.

real-time tools for the control of robots while executing remote manipulation tasks that are also subject to physical interaction uncertainties.

A preliminary implementation of this approach was presented by Ajoudani et al. (2015). The current study introduces fundamental improvements to the previous results by (i) implementing a novel human musculoskeletal model for more accurate tracking of the human arm kinematics; (ii) considering the effect of the muscle Jacobian to improve the contribution of arm kinematics and the synergistic contribution of muscle activities (at muscle level rather than joint level) to the arm endpoint stiffness; (iii) providing a more thorough description of the underlying principles and concepts; and (iv) evaluating the proposed controller for a realistic remote wall-drilling task with large dynamic physical interaction uncertainties.

\section{Reduced-complexity representation of the active arm endpoint stiffness}

According to Hill's musculoskeletal model, the stiffness of an individual muscle, $k_{m}(p, q)$, can be calculated from the stiffness values of the passive series element $k_{\mathrm{SE}}(q)$, the passive parallel element $k_{\mathrm{PE}}(q)$, and the contractile element $k_{\mathrm{CE}}(p)$, with $p$ and $q$ denoting the muscle activity and the joint angle vector, respectively. It is known that if the joint is far from its limits, where the musculotendon unit is not fully stretched or compressed, in the studies of active muscles, $k_{\mathrm{CE}}$ represents a fair estimate of the muscle stiffness, since $k_{\mathrm{SE}} \gg k_{\mathrm{CE}}$ and $k_{\mathrm{PE}} \ll k_{\mathrm{CE}}$ (Latash and Zatsiorsky, 1993).

The relation between the estimated active muscle stiffness $\left(\hat{K}_{m}\right)$ and the joint stiffness matrix $K_{J}$ can be calculated by

$$
K_{J}(p, q)=J_{m}^{\mathrm{T}}(q) \hat{K}_{m}(p) J_{m}(q)
$$

where ${ }^{\mathrm{T}}$ and $J_{m}$ are the transpose operator and the transformation relating musculotendon length changes to the joint angle variations. This matrix is computed by the partial derivative of the $i$ th muscle length $l_{i}$ with respect to the associating joint angles $q_{i} \in \mathbb{R}^{7}$ (Winters and Crago, 2012)

$$
J_{m}(q)=\left(\begin{array}{cccc}
\frac{\partial l_{1}(q)}{\partial q_{1}} & \frac{\partial l_{1}(q)}{\partial q_{2}} & \ldots & \frac{\partial l_{1}(q)}{\partial q_{n}} \\
\frac{\partial 2_{2}(q)}{\partial q_{1}} & \frac{\partial l_{2}(q)}{\partial q_{2}} & \ldots & \frac{\partial l_{2}(q)}{\partial q_{n}} \\
\vdots & \vdots & \ddots & \vdots \\
\frac{\partial l_{m}(q)}{\partial q_{1}} & \frac{\partial l_{m}(q)}{\partial q_{2}} & \ldots & \frac{\partial l_{m}(q)}{\partial q_{n}}
\end{array}\right)
$$

This relation assumes that no torques are present at the arm joints. Otherwise, the effect of joint torques on the joint stiffness variations must be included, based on the derivative of the muscle Jacobian with respect to the joint angles (Chen and Kao, 2000). While the configuration-dependent nature of the joint stiffness is mainly caused by the variations in passive joint properties, calculation of the Cartesian stiffness, i.e., $K_{c} \in \mathbb{R}^{6 \times 6}$, conversely, requires an additional transformation $J(q) \in \mathbb{R}^{6 \times 7}$, which is provided by the arm Jacobian (see next section) ${ }^{1}$

$$
K_{c}(p, q)=J^{+\mathrm{T}}(q)\left[K_{J}(p, q)-G_{J}(q)\right] J^{+}(q)
$$

For the given problem, we set the metric tensor $D=$ $K_{J}$ in the definition of the pseudo-inverse $J^{+}=D^{-1} J^{\mathrm{T}}$ $\left(J D^{-1} J^{\mathrm{T}}\right)^{-1}$ (Albu-Schaffer et al., 2004). The term $G_{J}(q)$ is defined by

$$
G_{J}(q)=\frac{\partial J^{\mathrm{T}}(q) f_{0}}{\partial q}+\frac{\partial \tau_{g}(q)}{\partial q}
$$

which captures the effect of arm geometry in the presence of external load $f_{0}$ and gravity $\tau_{g}(q)$. In this study, since the human arm does not interact with the external environment or carry a load, $f_{0}=0$. In addition, for the sake of simplicity in the identification of the human arm parameters, we neglect the effect of $\tau_{g}(q)$.

Traditionally, the active component of the estimated muscle stiffness matrix $\left(\hat{K}_{m}\right)$ is estimated using Hill's activation dynamic equations, which provide a mapping between the muscular activities, usually measured using EMG, and the corresponding muscles' stiffness profiles. This, however, requires that the EMG activities of several muscles are processed and passed through a complex system of equations to account for the muscle stiffness matrix, eventually resulting in costly (there is a need for several EMG sensors and amplification) and computationally intensive system (e.g., see Shin et al., 2009). Conversely, a dense literature gives solid evidence of the existence of synergistic relationships between arm mono- and bi-articular muscle activities, which realize a coordinated stiffening profile across the arm joints (Buchanan et al., 1986; Ison and Artemiadis, 2014a; Jamison and Caldwell, 1993; Kaneko et al., 2015; Osu and Gomi, 1999; Van Zuylen et al., 1988). As a result, cocontractions of the arm muscles mostly contribute to modifications in volume of the endpoint stiffness ellipsoid, rather than its direction (Ajoudani et al., 2012a). This strategy is deemed to be exploited by the central nervous system to solve for the motor complexity in an efficient and coordinated manner (Turvey, 2007). 
Therefore, we presume that (i) there exists a synergistic relationship between the arm muscle activities, and (ii) each muscle activation contributes to the volume of the endpoint stiffness ellipsoid with a different ratio. To clearly explain this choice, we can assume that the endpoint stiffness is represented by an ellipsoid, and that cocontractions modify the volume of such ellipsoid by changing the major axis lengths in a coordinated manner. Accordingly, we propose $\hat{K}_{m}=a_{c c}(p) K_{s}$, with $K_{s}$ an experimentally identified timeinvariant diagonal matrix (given that the muscle stiffness matrix is diagonal (Zatsiorsky, 2002)), which implements the contribution of each muscle to the active variations of the volume of the endpoint stiffness with a certain weight. The scalar and time-varying component, $a_{c c}(p)$, which is a function of the muscular activities, is multiplied by the muscle weights and computes the overall contribution of muscle activations to the volume of the endpoint stiffness ellipsoid. Obviously, depending on the choice of muscles that are used to compute the active component $\left(a_{c c}\right)$, the identified scale matrix $K_{s}$ would differ. In this study, as the most dominant and easily accessible muscles of the arm for surface electromyography measurements, we process the EMG activities of two muscles, i.e., the long heads of the biceps brachii $\left(P_{\mathrm{B}}\right)$ and triceps brachii $\left(P_{\mathrm{T}}\right)$, to calculate

$$
a_{c c}(p)=c_{1}+c_{2}\left(P_{\mathrm{B}}+P_{\mathrm{T}}\right)
$$

with $c_{1}$ and $c_{2}$ constant coefficients referring to the intrinsic muscle stiffness component ${ }^{2}$ and the active gain, which will be identified experimentally. By rearranging these equations, we obtain

$$
K_{c}(p, q)=J^{+\mathrm{T}}(q)\left[J_{m}^{\mathrm{T}}(q) a_{c c}(p) K_{s} J_{m}(q)-G_{J}(q)\right] J^{+}(q)
$$

It has been demonstrated that the configurationdependent effect of the muscle moment arm strongly depends on the muscle itself and the joint movement profile (e.g., flexion, extension, abduction, adduction). While this dependency can be significant for particular arm muscles and specific joint movements, on the contrary, the moment arm of those muscles and joint movements with a relatively trivial variation over the associating joint angles can be safely neglected (Ettema et al., 1998; Pigeon et al., 1996). Hence, in this paper, we exploit arm muscle length functions of eight dominant muscles acting on the shoulder and elbow joints, as described in Ettema et al. (1998) and Pigeon et al. (1996) to model moment arms of muscles as functions of the corresponding joint angles. The selected eight muscles are the anterior and posterior portions of the deltoid, the brachialis, the brachioradialis, the long and short portions of the biceps, and the long and lateral portions of the triceps, which present dominant effects in generation of the torque profiles in the arm joints.

\section{Human muscle and arm Jacobian tracking}

As shown in equation (6), the estimation of the endpoint stiffness $K_{c}$ is highly dependent on the tracking of the human arm configuration through two important Jacobians: the chain Jacobian, $J(q)$, and the muscle Jacobian, $J_{m}(q)$. Toward this objective, an online and easily scalable (subject-to-subject) model of the human right arm is developed that requires a minimum amount of sensory information to provide the two required Jacobians. This section presents a summary of the implementation of this model. ${ }^{3}$

The implementation of the proposed model is based on one of the human right arm models developed by the OpenSim Community, provided by Stanford University (Holzbaur et al., 2005). This model has seven degrees of freedom: three in the shoulder, two in the elbow, and two in the wrist. For the most part, OpenSim provides offline analysis of the human musculoskeletal model, since retrieval of the configuration of the human arm, i.e., a set of seven joint angles, from the original motion marker data is treated as a weighted least squares problem, which is actually a numerical optimization problem, trying to match the marker positions in the model to the corresponding experimental marker positions as accurately as possible (Hicks and Dembia, 2013). To make the estimation process of the human arm configuration available in real time, a compact, easyto-measure, and scalable representation of the human arm configuration, termed a generalized human arm triangle, is proposed and an analytical solution to the inverse kinematic problem from the generalized human arm triangle to the set of seven joint angles of the right arm is developed correspondingly. In comparison with the alternative descriptions, the generalized human arm triangle used in this study is able to express the arm posture in a more accurate way by considering the physiologically geometric constraints between segments and the whole skeleton model of human arm. Nevertheless, given the availability of the tracking systems and the target accuracy in real-time applications, alternative techniques for the real-time tracking of the arm and muscle Jacobians can be exploited (e.g., see Tolani and Badler, 1996).

The generalized human arm triangle is a dimensionindependent representation of the human arm configuration. It only requires a set of minimal orientation-related variables, which are easier to measure by commercially available motion capture systems than are position-related variables. The model consists of five variables, as shown in Figure 2, with the following parameters:

$r$ unit direction vector of the upper arm;

$l$ unit normal vector of the generalized human arm triangle plane. The direction of $l$ is determined by the right-hand rule, and the right-hand screw direction is the direction of elbow extension.

$\alpha$ angle between the upper arm and the lower arm;

$f$ unit direction vector of the fingers;

$p$ unit normal vector of the plane of the palm. Its direction points outward from the palm. 


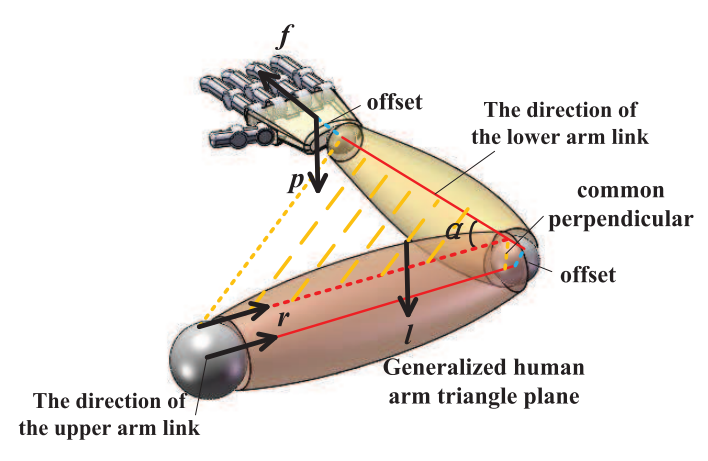

Fig. 2. Generalized human arm triangle model.

In this model, the links of the upper arm and the lower arm are non-coplanar. Hence, the generalized human arm triangle plane is defined as the plane determined by the lower arm line and the projection line of the upper arm line along their common perpendicular. There are actually seven independent variables in this model, owing to the normalization of the four vectors and the perpendicular relationships between the two pairs of vectors, $r$ versus $l$ and $f$ versus $p$. As a result, the human arm triangle space spanned by these five variables has a one-to-one relationship with the joint space spanned by the seven joint angle variables of the human arm kinematic model. This implies that the generalized human arm triangle model uniquely represents the arm configuration.

Conversely, the kinematic model of the human right arm, which is based on the modified Denavit-Hartenberg notation, can be retrieved from the original kinematic model file in OpenSim, the "Stanford VA Upper Limb Model". A diagram of the model is shown in Figure 3 and its corresponding Denavit-Hartenberg parameters are listed in Table 1 . The center of the base frame $\{0\}$ is located in the intersection point of the three rotational axes of shoulder joint. The $x$-axis of the last frame $\{T\}$ stands for the direction of thumb and the $z$ axis indicates the opposite direction of palm. All the body segments, to which the frames are attached, are shown in parentheses. It is worth noting that the dimension-related parameters, $l o_{\text {Humerus }}^{x}, l o_{\text {Humerus }}^{y}$, $l o_{\text {Humerus }}^{z}, l o_{\text {Ulna }}^{y}, l o_{\text {Ulna }}^{z}, l o_{\text {Radius }}^{y}, l o_{\text {Radius }}^{y^{\prime}}, l o_{\text {Radius }}^{a}, l o_{\text {Radius }}^{d}$, could be scaled to match human arms of different sizes (the trailing subscripts show the relevant body segments for scaling), which would make the model suitable for subjectto-subject variation. The analytical solution to the inverse kinematic problem from the generalized human arm triangle to the set of joint angles, $\theta_{i}(i=1,2, \ldots, 7)$, is developed in detail in (Fang et al., 2016) to capture and retrieve the arm configuration in an efficient way.

Once the configuration of the human arm has been reconstructed successfully, the relevant muscles around the human arm can be correspondingly located by utilizing the related muscle information from the model file (Holzbaur et al., 2005). Each elements of the muscle Jacobian $J_{m}(q)$,

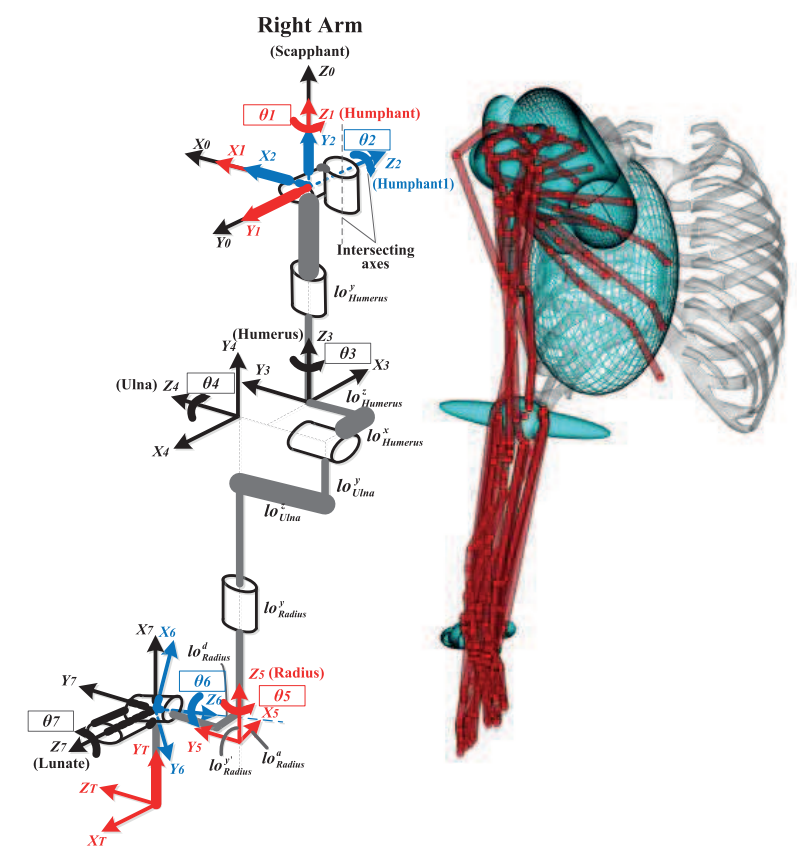

Fig. 3. Human right arm kinematic model in the modified Denavit-Hartenberg notation.

i.e., the muscle moment arms, are calculated as the derivative of the muscle length with respect to the corresponding joint angles (see equation (2)).

\section{Human arm endpoint stiffness estimation}

This section describes the procedure to identify the stiffness model parameters that correspond to the synergistic contributions of the chosen muscles $\left(K_{s}\right)$ to $K_{c}$, and the cocontraction index ( $c_{1}$ and $c_{2}$ in $\left.a_{c c}(p)\right)$.

To identify the model parameters, assuming that $J_{m}(q)$, $J(q)$, and $G_{J}(q)$ are calculated and known, $K_{c}$ in equation (6) must be identified in different configurations and cocontraction levels of the human arm. Following standard techniques for identification of the human arm endpoint stiffness in 3D (Perreault et al., 2002), stochastic perturbations were applied to the human arm endpoint and restoring forces were recorded using a six-axis force-torque sensor (ATI, Inc.), which was placed between the robot and the handle (see Figure 4). A KUKA lightweight robot IV was programmed in position control mode using Fast Research Interface (Schreiber et al., 2010), aimed at applying perturbation profiles to the arm endpoint through the handle. The amplitude of the applied perturbations had a peak-to-peak value of $20 \mathrm{~mm}$ in each direction. The frequency spectra of the perturbations were flat while decaying at a rate of $40 \mathrm{~dB} / \mathrm{Hz}$ at frequencies greater than $4 \mathrm{~Hz}$ (see Figure 5). This perturbation profile and the corresponding forces in response, ensure adequacy of data for the identification of endpoint dynamics (Mann et al., 1989). 
Table 1. Denavit-Hartenberg parameters of human right arm kinematic model.

\begin{tabular}{|c|c|c|c|c|}
\hline$i\left({ }^{i-1} T_{i}\right)$ & $\theta_{i}\left(^{\circ}\right)$ & $d_{i}(\mathrm{~m})$ & $a_{i-1}(\mathrm{~m})$ & $\alpha_{i-1}\left({ }^{\circ}\right)$ \\
\hline 1 & $\theta_{1}(0.0)$ & 0.00 & 0.00 & 0.0 \\
\hline 2 & $\theta_{2}(0.0)$ & 0.00 & 0.00 & 90.0 \\
\hline 3 & $\theta_{3}(-90.0)$ & $-l o_{\text {Humerus }}^{y}$ & 0.00 & -90.0 \\
\hline 4 & $\theta_{4}(180.0)$ & $l o_{U l n a}^{z}-1 o_{\text {Humerus }}^{z}$ & $-l o_{\text {Humerus }}^{x}$ & -90.0 \\
\hline 5 & $\theta_{5}(-124.2)$ & $-l o_{\text {Ulna }}^{y}-l o_{\text {Radius }}^{y}-l o_{\text {Radius }}^{y^{\prime}}$ & 0.00 & -90.0 \\
\hline 6 & $\theta_{6}(101.3)$ & $-l o_{\text {Radius }}^{d^{m}}$ & $l o_{\text {Radius }}^{a}$ & 97.8 \\
\hline 7 & $\theta_{7}(13.7)$ & 0.00 & 0.00 & -145.0 \\
\hline$T$ & -90.0 & 0.00 & -0.08 & -90.0 \\
\hline
\end{tabular}

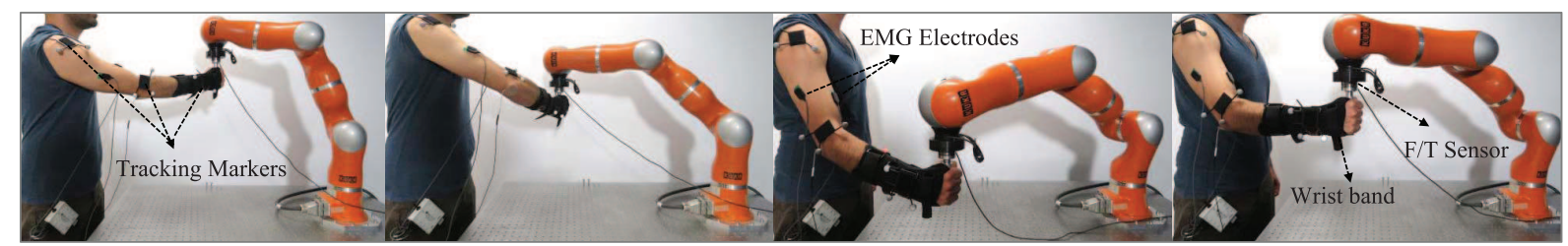

Fig. 4. Stiffness measurement experimental setup. A KUKA lightweight robot was programmed to apply stochastic perturbations to the human hand. Experiments were carried out in different arm configurations (typical examples are shown in this figure). Arm joints were allowed to vary within the redundant manifold of the corresponding shoulder and wrist position. Two subjects participated in the experiments.

$\mathrm{F} / \mathrm{T}$ : force-torque.

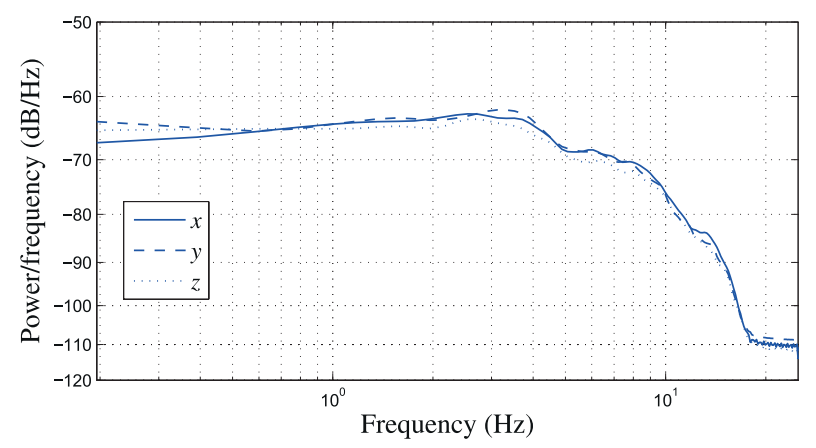

Fig. 5. Power spectrum of the applied position disturbances.

Experiments were carried out in seven different positions of the wrist with respect to the shoulder frame. These configurations were chosen anterior to the coronal plane of the body, within a reasonable workspace of the human arm while avoiding singular configurations and joint limits. In each set, arm joints were allowed to vary within the redundant manifold of the corresponding shoulder-wrist configuration to realize two or three distinct elevation angles of the shoulder joint (see Figure 4), resulting in 24 arm configurations in total. At each configuration, the subjects were asked to modulate and keep the cocontraction of the arm muscles in three different levels: minimum activity, medium activity $\left(20 \% P_{n_{\max }}\right)$, and high activity $\left(40 \% P_{n_{\max }}\right)$, where $P_{n_{\max }}$ is the maximum value of the cocontraction indicator and is calculated from the processed (acquired, filtered, and normalized at $1 \mathrm{kHz}$; see Ajoudani et al., 2012b, for details) EMG signals from two dominant, antagonistic upper arm muscles; namely the biceps $\left(P_{\mathrm{B}}\right)$ and triceps $\left(P_{\mathrm{T}}\right)$ brachii, hence

$$
P_{n}=\frac{P_{\mathrm{B}}+P_{\mathrm{T}}}{2}
$$

Throughout the experiments, $P_{n}$ was illustrated to the subject to keep the cocontraction levels as steady as possible. Meanwhile, body markers were attached to the human wrist, elbow, and shoulder for synchronized and precise tracking of (i) the human hand trajectories under perturbations and (ii) the human arm and muscle Jacobians, using an Optitrack motion tracking system at $100 \mathrm{~Hz}$. Acquisition, processing, control, and synchronization algorithms were all implemented in $\mathrm{C}++$.

Consequent to the acquisition and preprocessing of the position and restoring force trajectories of the human arm endpoint, multiple-input-multiple-output dynamics of the endpoint impedance were decomposed into the linear subsystems associating each input with each output (Ajoudani et al., 2012b). Based on this assumption, and indicating by $F_{x}(f), F_{y}(f)$, and $F_{z}(f)$ the Fourier transforms of the endpoint force along the axes of the Cartesian reference frame, with $x(f), y(f)$, and $z(f)$ the transforms of the human endpoint displacements, the dynamic relation between the displacements and force variations can be described by

$$
\left[\begin{array}{c}
F_{x}(f) \\
F_{y}(f) \\
F_{z}(f)
\end{array}\right]=\left[\begin{array}{lll}
G_{x x}(f) & G_{x y}(f) & G_{x z}(f) \\
G_{y x}(f) & G_{y y}(f) & G_{y z}(f) \\
G_{z x}(f) & G_{z y}(f) & G_{z z}(f)
\end{array}\right]\left[\begin{array}{c}
x(f) \\
y(f) \\
z(f)
\end{array}\right]
$$

A non-parametric algorithm was adopted to identify the empirical transfer function of each of the single-inputsingle-output (SISO) subsystems described previously in 
the frequency domain (MATLAB, The MathWorks Inc.). The smoothed spectral estimates of input and outputs (using windowing techniques) were fed to this algorithm in order to identify each SISO transfer function. Consequently, we adopted a parametric, second-order, linear model of each impedance transfer function of the type

$$
G_{i j}(s)=I_{c i j} \mathrm{~s}^{2}+B_{c i j} \mathrm{~s}+K_{c i j}, \quad \mathrm{~s}=2 \pi f \sqrt{-1}
$$

where $I_{c}, B_{c}$, and $K_{c}$ denote the endpoint inertia, viscosity, and stiffness matrices, respectively. The parameters of the second-order linear model were identified based on a least squares algorithm in the frequency range from 0 to $10 \mathrm{~Hz}$.

In a postprocessing stage, identified Cartesian stiffness matrices from the calibration trials were used to compute $K_{s}, c_{1}$, and $c_{2}$ by minimizing the Frobenius norm

$$
\left\|J_{m}^{\mathrm{T}}(q) a_{c c}(p) K_{s} J_{m}(q)-J^{\mathrm{T}}(q) K_{c}(p, q) J(q)\right\|
$$

with $J_{m} \in \mathbb{R}^{8 \times 7}, K_{s} \in \mathbb{R}^{8 \times 8}, J \in \mathbb{R}^{3 \times 7}$, and $K_{c} \in \mathbb{R}^{3 \times 3}$ (only translational components of the stiffness matrix). Considering that the muscle stiffness matrix is diagonal (Zatsiorsky, 2002), 10 unknown parameters $\left(K_{s}, c_{1}\right.$, and $\left.c_{2}\right)$ must be identified, which defines the minimum number of required trials for the calibration experiments. Based on this number, the total number of the trials was divided into the calibration and test trials for the validation of the identified model.

Once the model parameters have been identified, equation (6) can be utilized for the real-time computation of the arm endpoint stiffness profile using electromyography signals of one antagonistic pair of muscles and the tracking of the arm triangle.

\section{Results}

This section describes the results of the offline identification and real-time stiffness model for two healthy subjects (male, A (aged 33) and B (aged 32)).

\subsection{Identification experiments}

As stated before, experimental identification of the endpoint impedance matrices was performed by applying position perturbations and acquiring the force response within a $10 \mathrm{~Hz}$ frequency range. In all trials, multiple and partial coherence values of the force-position data (see Figure 6), positive definiteness, and symmetric ${ }^{4}$ measures of the estimated impedance matrices (Ajoudani et al., 2012b; Perreault et al., 2002) were the proving factors for the feasibility of the acquired results. Those trials that did not satisfy these conditions were discarded and repeated.

Figure 7 illustrates endpoint stiffness ellipsoids experimentally estimated from the perturbation experiments for three typical cocontraction profiles of the human arm, in three different configurations for subjects A (upper two rows) and B (lower row). For the sake of comparison, the 3D translational ellipsoids are projected to the $x y, x z$, and
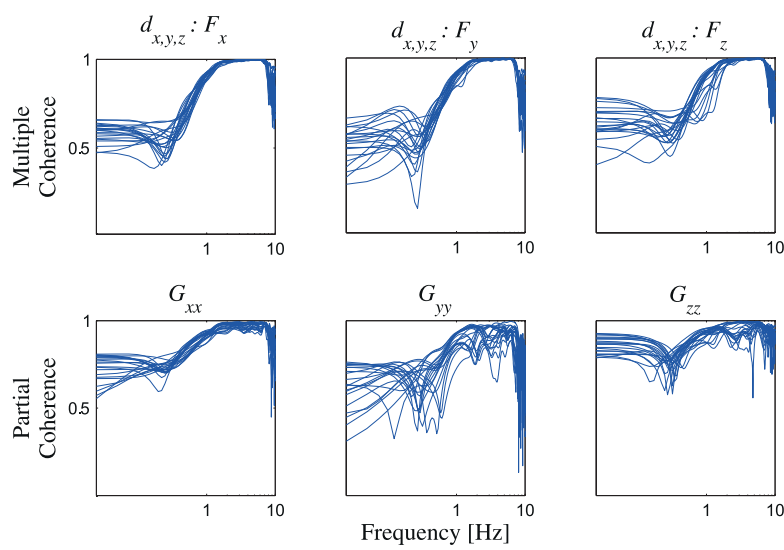

Fig. 6. Multiple and partial coherence values over the frequency range $\left[\begin{array}{ll}0 & 10\end{array}\right] \mathrm{Hz}$. These indexes investigate the linear dependency on each output to all system inputs, and between single input and single output, respectively. The $x$-axis scales are logarithmic and the $y$-axis scales are linear. Perfect coherence will be observed when the functions have values close to one. However, a linear approximation of the non-linear human arm endpoint stiffness behavior is naturally subject to a certain level of uncertainty, as observed in the plots.

$y z$ planes. Each ellipsoid's major axes directions and magnitudes are achieved from singular value decomposition of the corresponding projected stiffness matrix. These typical results show that the muscular cocontractions (minimum activity (green, solid), medium activity (blue, dashed), and high activity (red, dotted)) mostly contribute to the volume of the ellipsoid, with a negligible effect on its major axes directions (observable in each individual plot). Instead, a change of the arm configuration can substantially modify the geometry of the resulting ellipsoids, as observed in each row. It is worth remembering that no external forces are applied or received by the subjects.

To further investigate the effect of muscular cocontraction in volume adjustment of the endpoint stiffness ellipsoids, the correlation $\left(P<0.0005 ; R_{\mathrm{A}}{ }^{2}=0.76 ; R_{\mathrm{B}}{ }^{2}=\right.$ $0.89)$ between the cocontraction index $P_{n}$ and the volume index

$$
\Lambda_{K_{c}}^{\frac{1}{3}}=\left[\lambda_{1} \cdot \lambda_{2} \cdot \lambda_{3}\right]^{\frac{1}{3}}
$$

where $\lambda_{1}, \lambda_{2}$, and $\lambda_{3}$ are the eigenvalues of the corresponding experimentally identified endpoint stiffness matrix, is calculated for randomly selected trials and illustrated in Figure 8 . These figures illustrate a reasonably linear dependency between the cocontraction index and the volume index for subjects A (upper plot) and B (lower plot).

\subsection{Real-time stiffness model}

In a postprocessing phase, to evaluate the accuracy of the identified real-time model parameters, each subject's test trials $\left(n_{T}\right)$ were used to calculate the mean average error 

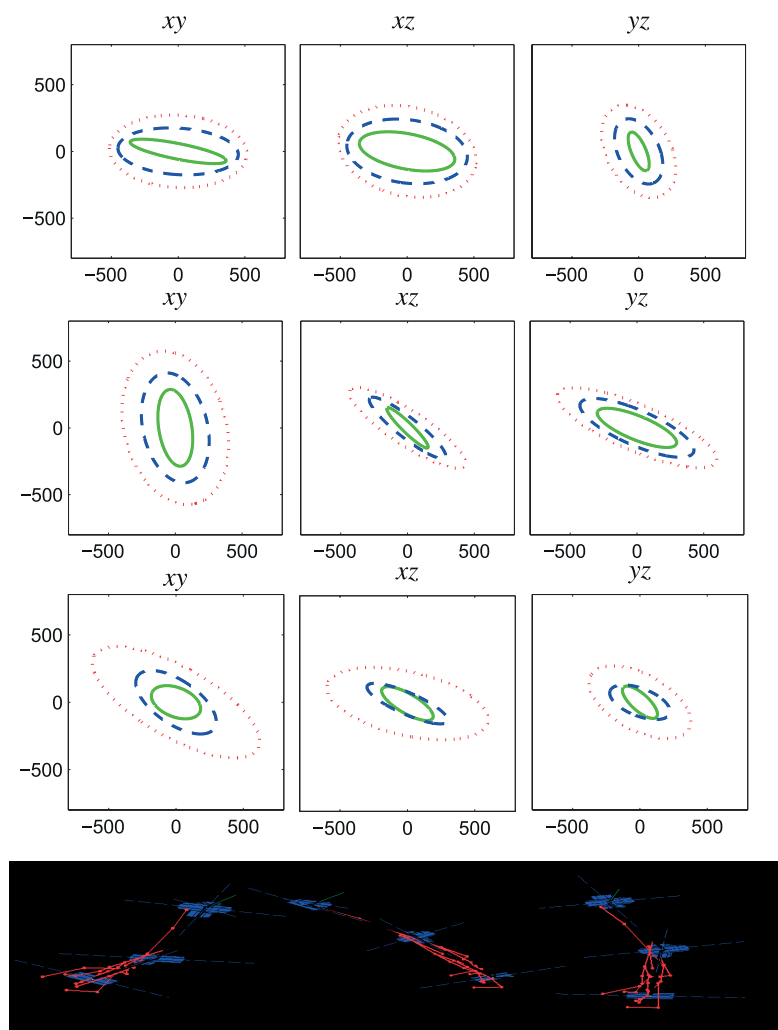

Fig. 7. Stiffness ellipsoids plotted for three distinct levels of the cocontraction values (minimum activity (green, solid), medium activity (blue, dashed), and high activity (red, dotted)) in three different arm configurations, illustrated from the top view in bottom plots. The numbers after the letter C- in the configurations refer to the row number in which the ellipsoids are plotted. The upper two rows are from subject A and the third from the top illustrates a typical example for subject B. Values are given in newtons per meter.

value between the results of the real-time model and the desired one using

$e_{K}=\frac{1}{n_{T}} \sum_{i=1}^{n_{T}} \frac{\left\|J_{m i}{ }^{\mathrm{T}}(q) a_{c c_{i}}(p) K_{s} J_{m i}(q)-J_{i}^{\mathrm{T}}(q) K_{c i}(p, q) J_{i}(q)\right\|}{\left\|K_{c i}(p, q)\right\|}$

which led to values of $12.3 \%$ and $11.5 \%$ for subjects $A$ and $\mathrm{B}$, respectively. Here, $K_{c i}(p, q)$ is the experimentally estimated endpoint stiffness matrix of the subject for trial $i$, and was used as the desired value for the real-time model that receives the arm configuration (through $J$ and $J_{m}$ ), $K_{s}$, and cocontraction index $\left(a_{c c}\right)$ to estimate the corresponding value. $^{5}$

It is important to note here that the stiffness modeling accuracy depends on the complexity of the model and the number of parameters to be identified. The main objective in this work was to achieve a reasonable trade-off between the two. This is because the number of unknown model parameters identifies the size of the experimental (training) data, which, owing to the complexity and difficulty of the stiffness estimation experiments, it is more practical to keep as low as possible. ${ }^{6}$ Hence, we neglected a part
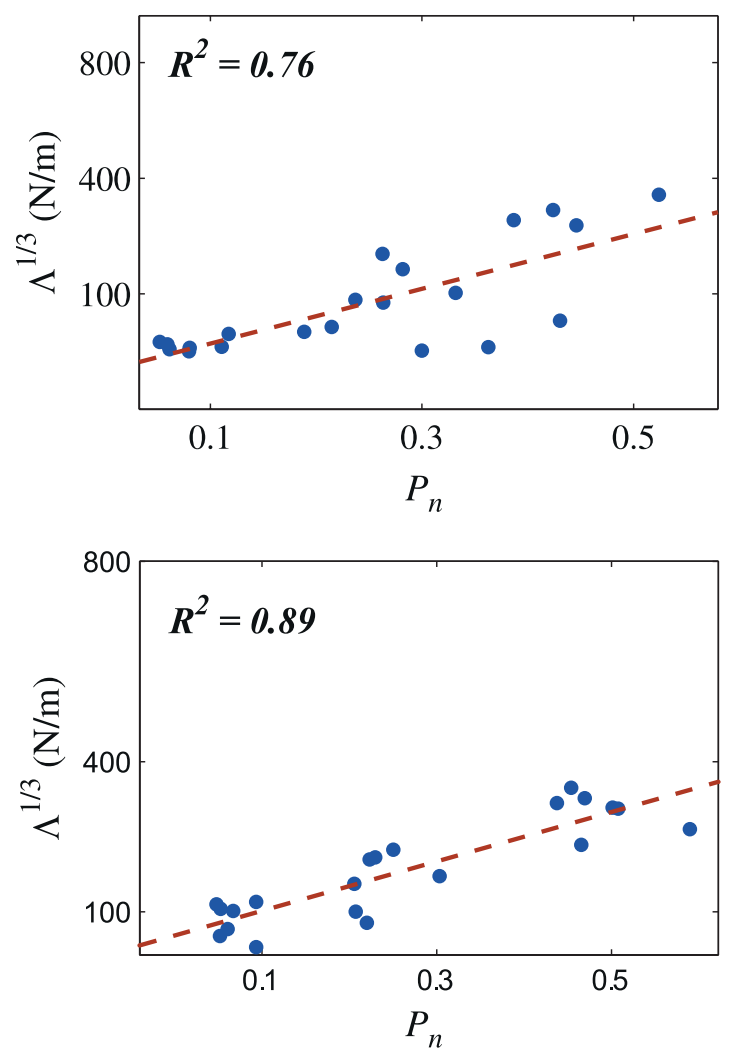

Fig. 8. Relationship between average muscular activities of the antagonistic pair $\left(P_{n}\right)$ and the volume index (cube root of $\Lambda$ ) of the experimentally identified endpoint stiffness matrices for subjects A (top) and B (bottom). These results support the use of linear mapping, $a_{c c}$, in this work.

of the complexity of the model that does not contribute to a substantial performance degradation (that lay within this $12 \%$ error range), and limited the number of unknown parameters to 10 .

Figure 9 illustrates typical results of the tracking of the human arm endpoint stiffness profile in different arm geometry and muscular cocontraction levels. Arm configurations (upper plots) of the master were tracked using the arm triangle data and used to estimate the arm joint angles and the two Jacobians. Estimated poses that include the muscle paths are calculated by our developed methods and visualized in the middle plots using MATLAB's robotic toolbox.

Computed arm Jacobians together with the cocontraction index $a_{c c}$ in two arbitrary contraction levels were used to calculate the Cartesian stiffness profiles along the $x$ $\left(K_{c x}\right), y\left(K_{c y}\right)$, and $z\left(K_{c z}\right)$ directions, in real time (lower plots). Results suggest that the chosen arm configurations have modulated the direction of the realized endpoint stiffness profile, proving that its volume can be adjusted using muscular cocontractions.

\section{Teleimpedance control experiments}

This section presents the experimental results of the proposed real-time model for the execution of remote tasks 


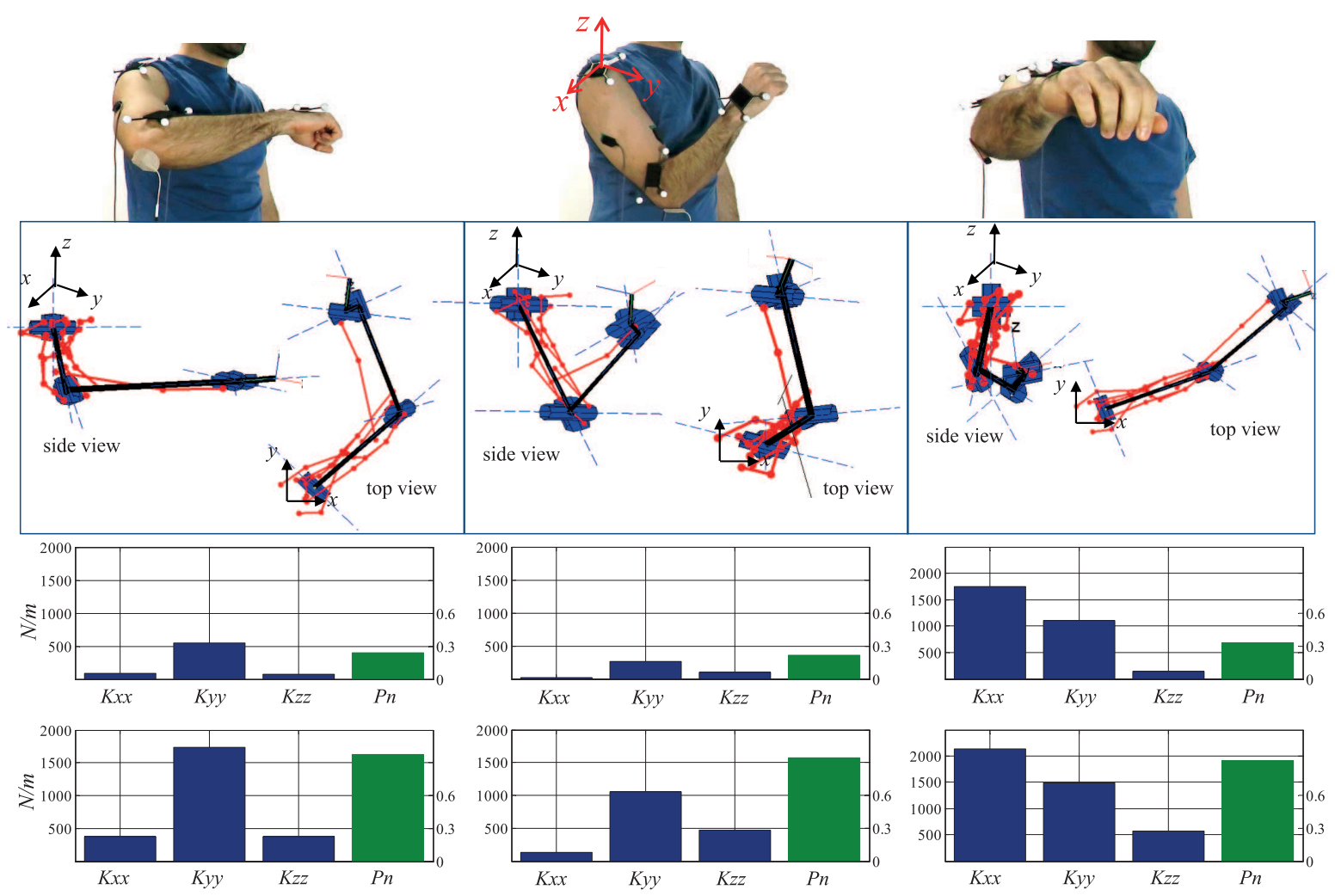

Fig. 9. Human configuration (top figures) and corresponding right arm musculoskeletal models (middle figures). Bottom plots illustrate results of real-time tracking of the human endpoint stiffness in three translational axes $\left(K_{x x}, K_{y y}\right.$, and $\left.K_{z z}\right)$, calculated from the human arm kinematics and EMG data $\left(P_{n}\right)$. The last two rows for each column correspond to different muscular cocontraction levels where the arm configuration is kept constant.

with dynamic uncertainties. As a representative example, we consider a remote wall-drilling task where the impedance planning and regulation of the tool play a dominant role in control of the interaction forces to accomplish the task: to penetrate the drill in a hard object, the force on the direction of the drill must be sufficiently high that it calls for a stiffer behavior in this direction.

Conversely, to avoid the generation of unnecessary interaction forces in remaining Cartesian directions, a relatively compliant behavior must be achieved so that if any undesired movement is generated by the operator, the interaction forces will remain reasonably low, so as not to damage the tool, the environment, or the robot. This behavior is way beyond the interaction capabilities of rigid robots or those operated in position control mode.

The experimental setup for the drilling task is illustrated in Figure 10. Two bricks are placed in different positions and orientations with respect to the KUKA base frame to evaluate the capability of the operator in adjustment of the hand trajectories and stiffness profile (volume and geometry) to perform the drilling task. The EMG signals (acquired at $1 \mathrm{kHz}$ ) and the arm kinematics (computed at $50 \mathrm{~Hz}$ ) are processed in real time and used to calculate the human arm endpoint stiffness according to equation (6). A Cartesian impedance controller is developed based on the study presented by Albu-Schaffer et al. (2003), to achieve the desired stiffness profile $\left(\in \mathbb{R}^{6 \times 6}\right)$ in real time. The rotational components of this matrix are considered constant, i.e., [70 7070 ] Nm/rad, while translational components replicate the operator's estimated endpoint stiffness matrix in real time. The Cartesian damping matrix is designed based on the desired Cartesian stiffness profile and the damping ratio $\xi=0.7$, according to Albu-Schaffer et al. (2003). Consequently, the computed joint torque profile is sent to the KUKA robot's torque controller through Fast Research Interface at $500 \mathrm{~Hz}$. All communication, processing, control, and synchronizations codes are developed in a $\mathrm{C}++$ environment.

The subject was asked to start from an arbitrary configuration (e.g., see Figure 11 left figure) and guide the robot end-effector to place the drill perpendicular to the brick on his left and perform the drilling task (see Figure 11 middle figure). Since the wrist kinematic data were used to control the KUKA end-effector movements, the subject was asked to use the shoulder and elbow configuration to control the drilling forces by geometric adjustment of his arm endpoint stiffness ellipsoid. If drilling forces were not sufficient and the drill was not penetrating into the rigid object, muscular coactivations could be used to increase the volume of the endpoint stiffness ellipsoid. This procedure was repeated 


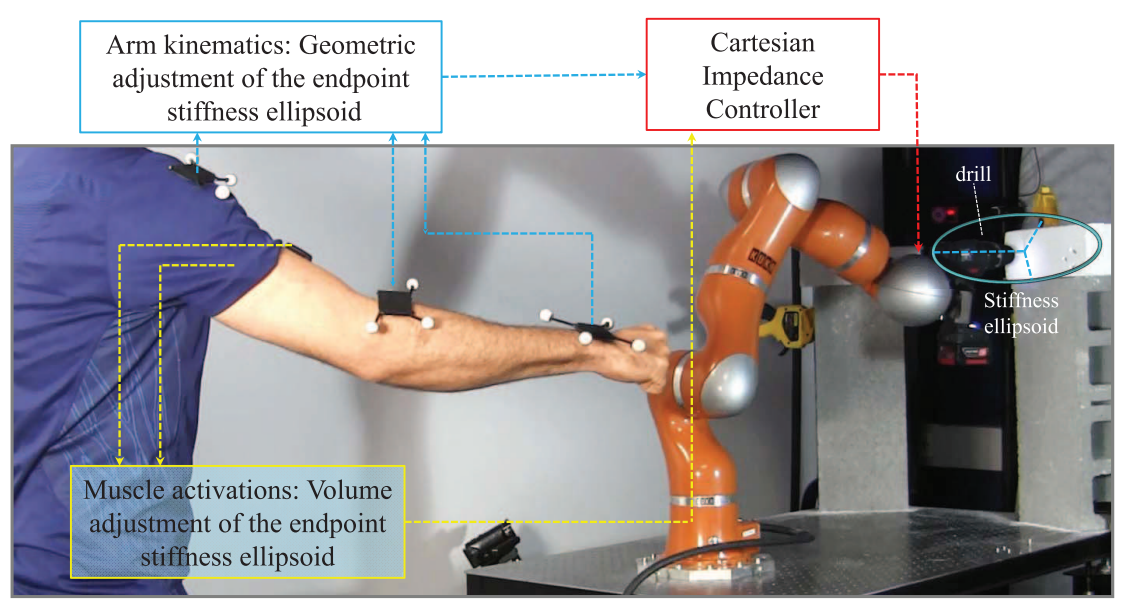

Fig. 10. Teleimpedance experimental setup. The muscular activities of the two antagonistic muscles are processed and used to compute the volume-adjusting component of the endpoint stiffness. The human arm kinematics (muscle paths, moment arms, arm Jacobian, etc.) are calculated from the three rigid body markers in real time and used to compute the geometry of the endpoint stiffness ellipsoid. The wrist tracking data are processed and used for the control of position and orientation of the robot end-effector trajectories in real time. The endpoint stiffness profile and the robot trajectories are achieved for the KUKA robot end-effector (drill) using an impedance controller developed here, which is based on the work of Albu-Schaffer et al. (2003).

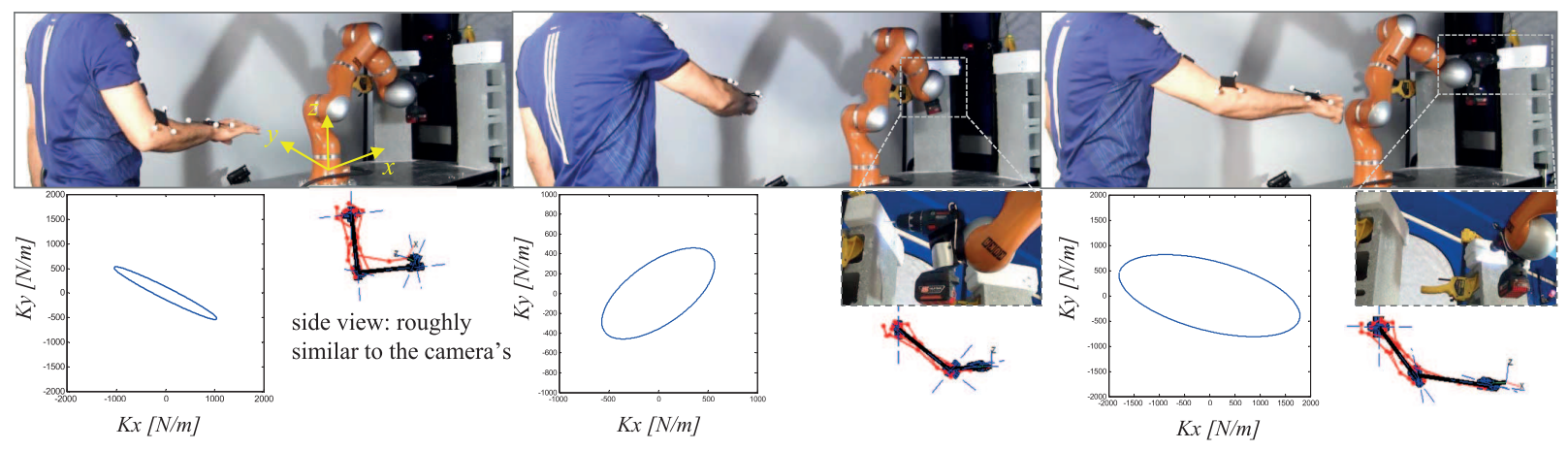

Fig. 11. Selected scenes from the wall-drilling experiment (see video attachment). Starting from an initial configuration (most left figures), the operator adjusts the robot end-effector position and orientation to place the drill vertically on the brick on his left, while adjusting the arm configuration (shoulder and elbow) to increase the stiffness on the drilling direction (middle figures). The procedure is repeated for the right side wall, as observed in the most right figures. The stiffness ellipsoids in $x y$ plane that correspond to a particular arm configuration and muscular activity level (see Fig.12) are plotted at the bottom of the selected scene. The ellipsoids are plotted in KUKA frame, which is illustrated using the yellow arrows.

for the brick on the operator's right side, as illustrated in the pose on the right of Figure 11. The subject had complete visual perception of the scene, since the robot, the bricks, and the operator were all located in the same room.

The operators processed EMG signals (filtered and normalized); the estimated endpoint stiffness profiles in the three main Cartesian directions are plotted in Figure 12. The stiffness ellipsoids in the $x y$ plane that correspond to a particular arm configuration and muscular activity levels (A, $\mathrm{B}$, and C) are plotted at the bottom of the selected scenes of Figure 11. The ellipsoids are plotted in the KUKA frame, as illustrated by the yellow arrows in Figure 11 .

These results illustrate that the master is capable of modulating the task forces using selected arm poses and the coactivation levels of the arm muscles. The achieved arm pose of the human at each phase orients the end-effector stiffness in such a way that the major axis, with a stiffer profile, coincides with the drilling direction. The remaining axes instead achieve a more compliant behavior to reduce the effect of involuntary movement of the human wrist in the generation of unnecessary interaction forces.

\section{Conclusions}

In this paper, with the purpose of achieving a desired physical interaction performance in a teleimpedance control setup, a novel, computationally efficient, and real-time model of the human arm endpoint stiffness was proposed. 

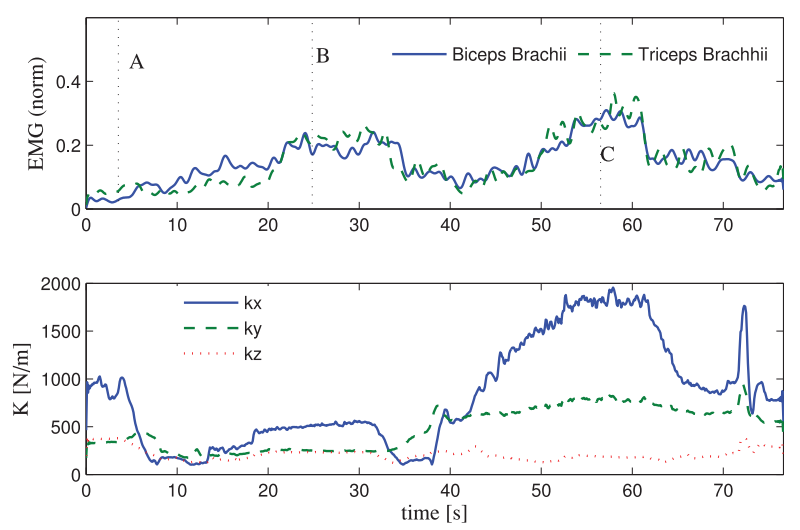

Fig. 12. Processed (filtered and normalized) electromyography (EMG) signals of the biceps brachii and triceps brachii muscles are illustrated in the upper plot during the teleimpedance experiment. The resulting human arm endpoint stiffness (achieved in KUKA endpoint using the Cartesian impedance controller of the robot), in KUKA frame of reference is illustrated in the lower plot. Lines, A, B, and C roughly correspond to the left, middle, and right configurations of Figure 11.

Toward this end, a real-time model of the human arm kinematics, including muscle paths, arm Jacobian, etc., was introduced and used for the computation of the dominant effect of arm kinematics in geometric modifications of the arm endpoint stiffness. In addition, a cocontraction index was defined using muscular activities of a dominant antagonistic muscle pair, which contributes to a coordinated stiffening in all axes of the endpoint stiffness ellipsoid. The calibration and identification of the model parameters were carried out experimentally, using perturbation-based arm endpoint stiffness measurements in different arm configurations and cocontraction levels of the chosen muscles. The proposed experimental study, which concerned the drilling of hard objects placed in different positions and orientations of the robot workspace, evaluated the proposed model in the generation of a desired stiffness profile, in real time. Results suggested that the proposed model provided the master with the ability to regulate the direction of the principal axes of the endpoint stiffness ellipsoid and its volume by choosing the arm pose and applying muscular cocontractions, respectively.

As mentioned previously, any reduced-complexity representation of the highly complex human neuromotor system is certainly subject to modeling uncertainties and inaccuracies. For example, the contractile element $k_{\mathrm{CE}}$ depends not only on the activation of the muscle but also on the length of the muscle itself, which is non-linearly related to the joint angle pose and velocity (Piovesan et al., 2013). Furthermore, for muscles with long tendons or in elderly individuals, the relation $k_{\mathrm{SE}} \gg k_{\mathrm{CE}}$ and $k_{\mathrm{PE}} \ll k_{\mathrm{CE}}$ may not be valid (Piovesan et al., 2011).

It is important to note that some previous work (Lacquaniti et al., 1993; Burdet et al., 2001; Franklin et al., 2007) has illustrated that human beings can learn to change the orientation of the endpoint stiffness ellipsoid toward the direction of the external disturbance. However, these studies did not address the extent of the stiffness modulation in constant arm poses, which has been quantitatively shown to be limited in other work (Perreault et al., 2002; Trumbower et al., 2009). In particular, Perreault et al. (2002) demonstrated that the range of available stiffness orientations decreases as endpoint force exertion increases, ${ }^{7}$ and the endpoint force direction significantly constrains direction and magnitude of the stiffness orientations that can be achieved. This range was reported to be only $0.91^{\circ} \pm 5.85^{\circ}$. Given these findings, Perreault et al. (2002) claim that it appears unlikely that static endpoint stiffness orientation is controlled independently of force by voluntary neural mechanisms during postural tasks.

Future work will focus on the integration of wearable tactile feedback or model-mediated cutaneous force feedback (Mitra and Niemeyer, 2008) to our teleimpedance setup for improved transparency, while guaranteeing the robustness and stability of the teleoperation interface.

\section{Funding}

The author(s) disclosed receipt of the following financial support for the research, authorship, and/or publication of this article: This work was supported in part by the European projects CogIMon (grant number H2020 ICT-23-2014 644727), WALKMAN (grant number EU FP7-ICT 611832), and SoftPro (grant number H2020 ICT 688857).

\section{Notes}

1. In this study, we neglect the effect of reflex feedback in muscle stiffness variations.

2. It is worth mentioning that the main focus of this study is to determine the active stiffness of the human limb; thus, the passive modeling (including tendon and muscle passivity) is subject to uncertainty. Especially concerning special cases, e.g., in elderly subjects, the tendon stiffness is likely to increase. Future research will be conducted to evaluate the estimated accuracy of the passive components.

3. The new kinematic model improves the accuracy of the joint angle identification and the arm Jacobian calculation previously presented by Fang and Ding (2013). In addition, it provides muscles paths as a function of the joint angles that are used for the calculation of moment arms, i.e., the muscle Jacobian, in real time. While avoiding replication of the content of the kinematic model published elsewhere (Fang et al., 2016), in this paper, we aim to provide enough detail and evidence to support the accuracy of the proposed model.

4. By calculating $K_{c}-K_{c}^{\mathrm{T}} / K_{c}+K_{c}^{\mathrm{T}}$, which resulted in an average value of $9 \%$ overall trials for subjects A and B.

5. Results of the identification of $K_{S}$ for subjects A [3.8509, $18.8039,7.0586,4.8555,0.8714,0.0450,9.2425,5.3625]$ and B $[2.3006,11.2550,4.3380,2.9111,0.2019,0.5832,5.6096$, 1.4676] demonstrate a good consistency between the two. The scales are normalized to the parameter $c_{2}$ in equation (5).

6. This limitation is probably the main reason for a limited performance of non-parametric or model free approaches in 
modeling human dynamics (which are usually not directly measurable) that require a rich training dataset.

7. Consider that force production in human beings is accompanied by the coactivation of the involved antagonistic muscles, but the reverse may not be true (Gribble et al., 2003).

\section{References}

Ajoudani A (2016) Transferring Human Impedance Regulation Skills to Robots. Cham: Springer.

Ajoudani A, Fang C, Tsagarakis NG, et al. (2015) A reduced-complexity description of arm endpoint stiffness with applications to teleimpedance control. In: 2015 IEEE/RSJ international conference on intelligent robots and systems (IROS), Hamburg, Germany, 28 September-2 October 2015, pp. 1017-1023. Piscataway, NJ: IEEE.

Ajoudani A, Gabiccini M, Tsagarakis N, et al. (2012a) TeleImpedance: Exploring the role of common-mode and configuration-dependant stiffness. In: 12th IEEE-RAS international conference on humanoid robots (humanoids), Osaka, Japan, 29 November-1 December 2012, pp. 363-369. Piscataway, NJ: IEEE.

Ajoudani A, Tsagarakis N, and Bicchi A (2012b) Tele-impedance: Teleoperation with impedance regulation using a bodymachine interface. International Journal of Robotics Research 31(13): 1642-1655.

Akazawa K, Milner TE, and Stein RB (1983) Modulation of reflex EMG and stiffness in response to stretch of human finger muscle. Journal of Neurophysiology 49(1): 16-27.

Albu-Schaffer A, Fischer M, Schreiber G, et al. (2004) Soft robotics: What Cartesian stiffness can obtain with passively compliant, uncoupled joints? In: IEEE/RSJ international conference on intelligent robots and systems (IROS), Sendai, Japan, 28 September-2 October 2004, vol. 4, pp. 3295-3301. Piscataway, NJ: IEEE.

Albu-Schaffer A, Ott C, Frese U, et al. (2003) Cartesian impedance control of redundant robots: Recent results with the DLR-light-weight-arms. In: Proceedings. ICRA'03. IEEE international conference on robotics and automation, Taipei, Taiwan, 14-19 September 2003, vol. 3, pp. 3704-3709. Piscataway, NJ: IEEE.

Buchanan TS, Almdale D, Lewis JL, et al. (1986) Characteristics of synergic relations during isometric contractions of human elbow muscles. Journal of Neurophysiology 56(5): 1225-1241.

Burdet E, Osu R, Franklin D, et al. (2001) The central nervous system stabilizes unstable dynamics by learning optimal impedance. Nature 414(6862): 446-449.

Castellini C, Artemiadis P, Wininger M, et al. (2014) Proceedings of the first workshop on peripheral machine interfaces: Going beyond traditional surface electromyography. Frontiers in Neurorobotics 8: PMC4133701.

Chen SF and Kao I (2000) Conservative congruence transformation for joint and Cartesian stiffness matrices of robotic hands and fingers. The International Journal of Robotics Research 19(9): 835-847.

Ettema G, Styles G, and Kippers V (1998) The moment arms of 23 muscle segments of the upper limb with varying elbow and forearm positions: Implications for motor control. Human Movement Science 17(2): 201-220.

Fang C and Ding X (2013) A set of basic movement primitives for anthropomorphic arms. In: 2013 IEEE international conference on mechatronics and automation (ICMA), Takamatsu, Japan, 4-7 August 2013, pp. 639-644. Piscataway, NJ: IEEE.

Fang C, Ajoudani A, Tsagarakis NG, et al. (2016) An analytical identification and tracking method of the kinematic musculoskeletal model of human right arm for real-time applications. IEEE Transactions on Biomedical Engineering 22(6): $1216-1227$.

Franklin DW, Burdet E, Osu R, et al. (2003) Functional significance of stiffness in adaptation of multijoint arm movements to stable and unstable dynamics. Experimental Brain Research 151(2): 145-157.

Franklin DW, Liaw G, Milner TE, et al. (2007) Endpoint stiffness of the arm is directionally tuned to instability in the environment. Journal of Neuroscience 27(29): 7705-7716.

Gribble PL, Mullin LI, Cothros N, et al. (2003) Role of cocontraction in arm movement accuracy. Journal of Neurophysiology 89(5): 2396-2405.

Hannaford B and Ryu JH (2002) Time-domain passivity control of haptic interfaces. IEEE Transactions on Robotics and Automation 18(1): 1-10.

Hicks J and Dembia C (2013) How inverse kinematics works in OpenSim. Available at: https://simtk-confluence.stanford. edu:8443/display/OpenSim/How+Inverse+Kinematics+Works\# HowInverseKinematicsWorks-_Toc174781343WeightedLeast Squares Equation (accessed 5 March 2016).

Hocaoglu E and Patoglu V (2012) Tele-impedance control of a variable stiffness prosthetic hand. In: IEEE international conference on robotics and biomimetics (ROBIO), Guangzhou, China, 11-14 December 2012, pp. 1576-1582. Piscataway, NJ: IEEE.

Holzbaur KR, Murray WM, and Delp SL (2005) A model of the upper extremity for simulating musculoskeletal surgery and analyzing neuromuscular control. Annals of Biomedical Engineering 33(6): 829-840.

Howard M, Braun DJ, and Vijayakumar S (2013) Transferring human impedance behavior to heterogeneous variable impedance actuators. IEEE Transactions on Robotics 29(4): 847-862.

Ison M and Artemiadis P (2014a) Proportional myoelectric control of robots: Muscle synergy development drives performance enhancement, retainment, and generalization. IEEE Transactions on Robotics 31(2): 259-268.

Ison $\mathrm{M}$ and Artemiadis $\mathrm{P}(2014 \mathrm{~b})$ The role of muscle synergies in myoelectric control: Trends and challenges for simultaneous multifunction control. Journal of Neural Engineering 11(5): 051001.

Jamison JC and Caldwell GE (1993) Muscle synergies and isometric torque production: Influence of supination and pronation level on elbow flexion. Journal of Neurophysiology 70(3): 947-960.

Kaneko T, Ogata K, Sakaino S, et al. (2015) Impact force control based on stiffness ellipse method using biped robot equipped with biarticular muscles. In: 2015 IEEE/RSJ international conference on intelligent robots and systems (IROS), Hamburg, Germany, 28 September-2 October 2015, pp. 2246-2251. Piscataway, NJ: IEEE.

Kawato M (1999) Internal models for motor control and trajectory planning. Current Opinion in Neurobiology 9(6): 718-727.

Lacquaniti F, Carrozzo M, and Borghese N (1993) Time-varying mechanical behavior of multijointed arm in man. Journal of Neurophysiology 69(5): 1443-1464. 
Latash ML and Zatsiorsky VM (1993) Joint stiffness: Myth or reality? Human Movement Science 12(6): 653-692.

Lloyd DG and Besier TF (2003) An EMG-driven musculoskeletal model to estimate muscle forces and knee joint moments in vivo. Journal of Biomechanics 36(6): 765-776.

Mann K, Werner F, and Palmer A (1989) Frequency spectrum analysis of wrist motion for activities of daily living. Journal of Orthopaedic Research 7: 304-306.

Milner TE (2002) Contribution of geometry and joint stiffness to mechanical stability of the human arm. Experimental Brain Research 143(4): 515-519.

Mitra P and Niemeyer G (2008) Model-mediated telemanipulation. The International Journal of Robotics Research 27(2): 253-262.

Mussa-Ivaldi FA, Hogan N, and Bizzi E (1985) Neural, mechanical, and geometric factors subserving arm posture in humans. Journal of Neuroscience 5(10): 2732-2743.

Osu R and Gomi H (1999) Multijoint muscle regulation mechanisms examined by measured human arm stiffness and EMG signals. Journal of Neurophysiology 81(4): 1458-1468.

Perreault EJ, Kirsch RF, and Crago PE (2002) Voluntary control of static endpoint stiffness during force regulation tasks. Journal of Neurophysiology 87(6): 2808-2816.

Pigeon P, Yahia L, and Feldman AG (1996) Moment arms and lengths of human upper limb muscles as functions of joint angles. Journal of Biomechanics 29(10): 1365-1370.

Piovesan D, Pierobon A, DiZio P, et al. (2011) Comparative analysis of methods for estimating arm segment parameters and joint torques from inverse dynamics. Journal of Biomechanical Engineering 133(3): 031003.

Piovesan D, Pierobon A, DiZio P, et al. (2013) Experimental measure of arm stiffness during single reaching movements with a time-frequency analysis. Journal of Neurophysiology 110(10): 2484-2496.

Schreiber G, Stemmer A, and Bischoff R (2010) The fast research interface for the KUKA lightweight robot. In: IEEE workshop on innovative robot control architectures for demanding (research) applications how to modify and enhance commercial controllers (ICRA 2010), Anchorage, AK, 3-8 May 2010, pp. 15-21. Piscataway, NJ: IEEE.
Selen LP, Beek PJ, and van Dieën JH (2005) Can co-activation reduce kinematic variability? A simulation study. Biological Cybernetics 93(5): 373-381.

Shin D, Kim J, and Koike Y (2009) A myokinetic arm model for estimating joint torque and stiffness from EMG signals during maintained posture. Journal of Neurophysiology 101(1): $387-401$

Tolani D and Badler NI (1996) Real-time inverse kinematics of the human arm. Presence: Teleoperators \& Virtual Environments 5(4): 393-401.

Trumbower RD, Krutky MA, Yang BS, et al. (2009) Use of self-selected postures to regulate multi-joint stiffness during unconstrained tasks. PLOS ONE 4(5): e5411.

Turvey MT (2007) Action and perception at the level of synergies. Human Movement Science 26(4): 657-697.

Van Zuylen E, Gielen C, and Van Der Gon JD (1988) Coordination and inhomogeneous activation of human arm muscles during isometric torques. Journal of Neurophysiology 60(5): $1523-1548$.

Winters JM and Crago PE (2012) Biomechanics and Neural Control of Posture and Movement. New York: Springer.

Zatsiorsky VM (2002) Kinetics of Human Motion. Champaign, IL: Human Kinetics.

\section{Appendix: Index to multimedia extension}

Archives of IJRR multimedia extensions published prior to 2014 can be found at http://www.ijrr.org, after 2014 all videos are available on the IJRR YouTube channel at http://www.youtube.com/user/ijrrmultimedia

\section{Table of multimedia extension.}

\begin{tabular}{lll}
\hline Extension & Media type & Description \\
\hline 1 & Video & Video of the drilling experiment \\
\hline
\end{tabular}

